\title{
Resiliência em cuidadores familiares de idosos com doença de Alzheimer
}

\author{
Carlene Souza Silva Manzini ${ }^{1}$, Francisco Assis Carvalho Vale ${ }^{2}$
}

\author{
${ }^{1}$ Enfermeira, Mestre em Enfermagem. \\ Discente do Programa de Pós-Graduação \\ em Ciências da Saúde, nível Doutorado, da \\ Universidade Federal de São Carlos. São \\ Carlos, SP, Brasil. E-mail: \\ carlotamanzi@hotmail.com. \\ ${ }^{2}$ Médico Neurologista, Doutor em \\ Neurologia. Professor Adjunto da \\ Universidade Federal de São Carlos. São \\ Carlos, SP, Brasil. E-mail: \\ facvale@ufscar.br.
}

Recebido: 22/08/2015.

Aceito: 02/09/2016.

Publicado: 12/12/2016

\section{Como citar esse artigo:}

Manzini CSS, Vale FAC. Resiliência em cuidadores familiares de idosos com doença de Alzheimer. Rev. Eletr. Enf. [Internet]. 2016 [acesso em:

____18:e1190. Disponível em: http://dx.doi.org/10.5216/ree.v18.37035.

\begin{abstract}
RESUMO
Resiliência é uma capacidade das pessoas de enfrentar de forma positiva as adversidades. $O$ estudo transversal, quantitativo, teve por objetivo avaliar fatores associados à resiliência em cuidador familiar de idosos com doença de Alzheimer. A amostra foi composta por 66 cuidadores de idosos, acompanhados em um ambulatório de neurologia. A maioria dos cuidadores apresentou resiliência moderada. Análise de regressão linear mostrou que alguns fatores interferem na resiliência dos cuidadores sendo estes: sobrecarga, aumento do número de dias dedicados ao cuidado, grau de parentesco nora/genro ou cônjuge, e prática de outras atividades além do cuidado. Os achados poderão ser úteis em áreas ligadas ao cuidado, e uma vez que foram identificadas variáveis que interferem na resiliência, estas poderão ser trabalhadas e melhoradas em benefício do cuidador e do paciente.

Descritores: Resiliência Psicológica; Doença de Alzheimer; Cuidadores; Demência; Família.
\end{abstract}

\section{INTRODUÇÃO}

A demência é um problema de saúde frequente e acarreta enorme custo socioeconômico. As estimativas de prevalência na maior parte do mundo variam entre $5 \%$ a $7 \%$, chegando a $85 \%$ na América Latina $^{(1)}$

Demência é uma síndrome caracterizada por sintomas cognitivos ou comportamentais (sintomas neuropsiquiátricos) que interferem no trabalho ou outras atividades da vida diária e representam um declínio de um nível prévio de funcionalidade, que não são explicadas por delirium ou transtornos psiquiátricos graves $^{(2)}$.

Existem vários tipos de demência, porém a advinda da doença de Alzheimer (DA) é a mais prevalente, 
acometendo de 50 a $60 \%$ dos casos, sendo uma das principais causas de incapacidade na vida adulta ${ }^{(3)}$. Diante desse cenário surge o papel do cuidador, na medida em que aparecem as dificuldades cotidianas de uma nova realidade, exigindo a tomada de decisões e a incorporação de atividades que passam a ser de sua inteira responsabilidade.

Praticar e manter o cuidado a um familiar com doença crônica, sobretudo progressiva e neurodegenerativa como a DA, é algo que requer boa saúde física e mental do cuidador, uma vez que as demandas por parte do doente exigem um ritmo crescente de atenção e cuidado, conforme se aceleram os estágios da doença.

Cuidador é o indivíduo que, no espaço privado doméstico, realiza ou ajuda a pessoa com limitação a realizar suas atividades básicas e instrumentais de vida diária (desde a higiene pessoal até a administração financeira da família), com o objetivo da preservação de sua autonomia e de sua independência ${ }^{(4)}$.

A tarefa de cuidar de um familiar com demência é reconhecida como associada a problemas físicos e emocionais no cuidador $^{(5)}$. O desgaste da rotina de cuidados pode ocorrer devido à falta de apoio aos cuidadores, falta de conhecimento destes sobre os estágios da demência e falta de preparo para o cuidado.

No intuito de amenizar a sobrecarga física e emocional, os cuidadores familiares necessitariam desenvolver atitudes positivas, que os ajudassem a suportar a gama de fatores negativos e nocivos à saúde advinda do processo de cuidar. $\mathrm{O}$ desenvolvimento de maiores níveis de resiliência poderia vir a beneficiar tanto o cuidador como o idoso que está sob seus cuidados.

Como capacidade de todo ser humano de adaptar-se recorrendo a recursos internos e externos, a resiliência pode ser compreendida como uma manutenção de um processo de desenvolvimento, apesar das condições difíceis, sendo algo sistemático, dinâmico e complexo, resultando da interação entre o indivíduo e o meio ${ }^{(6)}$.

Ao focar na resiliência como capacidade humana, emerge uma das possibilidades de redirecionar os cuidados da saúde para reconhecer as forças e desenvolver estratégias para construir capacidades existentes, já que a resiliência conota em força interior, competência, otimismo, flexibilidade e habilidade para lidar de forma efetiva com a adversidade ${ }^{(7)}$. Dessa forma, conhecer fatores capazes de influenciar no processo de manutenção da resiliência, pode auxiliar o enfermeiro na instrumentalização, capacitação e formação de sua equipe, para que esta possa fortalecer as potencialidades, promover e estimular novas formas de enfrentamento das dificuldades, sobretudo daqueles indivíduos que apresentarem de moderada a baixa resiliência.

O objetivo deste estudo foi avaliar fatores associados à resiliência de cuidador familiar de idosos com doença de Alzheimer.

\section{MATERIAL E MÉTODOS}

Trata-se de estudo de corte transversal, descritivo, correlacional e de caráter quantitativo.

A pesquisa foi realizada no Ambulatório de Neurologia Cognitiva e Comportamental da UFSCar (ANEU), 
sediado no Centro Municipal de Especialidades (CEME), no município de São Carlos - SP, no período de maio a dezembro de 2014. O ANEU é um ambulatório médico neurológico especializado, que atende pessoas com transtornos cognitivos e comportamentais associados a doenças neurológicas e não neurológicas, destacando-se as demências.

Foram incluídos no estudo cuidadores familiares que conviviam e cuidavam diariamente do idoso, que preferencialmente residiam no mesmo domicílio, que estavam na função de cuidador há um ano ou mais, maiores de 18 anos, de ambos os sexos, qualquer escolaridade.

A amostra foi composta por cuidadores familiares de idosos diagnosticados com DA, que eram acompanhados no Ambulatório de Neurologia da UFSCar (ANEU). As fases da demência foram constatadas por meio de escala de Estagiamento Clínico de Demência, Clinical Dementia Rating (CDR). O tamanho da amostra foi de 66 sujeitos, distribuídos conforme o estágio da doença. Grupo DA - 66 cuidadores familiares, distribuídos em três subgrupos conforme o estágio da demência:

a) Subgrupo DA leve - 25 cuidadores, os idosos com demência na fase leve.

b) Subgrupo DA moderada - 22 cuidadores, os idosos com demência na fase moderada.

c) Subgrupo DA avançada - 19 cuidadores, os idosos com demência na fase avançada.

Após aprovação do projeto pelo Comitê de Ética em Pesquisa em Seres Humanos da Universidade Federal de São Carlos - UFSCar, Parecer consubstanciado no 489.795, solicitou-se autorização para coleta de dados aos coordenadores do Centro de Especialidades Médicas (CEME). Os cuidadores que se encaixavam nos critérios de inclusão, eram convidados a fazer parte da pesquisa, assinando o Termo de Consentimento Livre e Esclarecido (TCLE).

Para a coleta dos dados foram utilizados os seguintes instrumentos: Questionário de Caracterização do Cuidador: elaborado pelos pesquisadores, que permitia acesso a informações pessoais, hábitos de vida e características do cuidar; Critério de Classificação Econômica Brasil (CCEB): instrumento utilizado para estimar o poder de compra das famílias e pessoas urbanas. Escala de Resiliência (ER): um dos poucos instrumentos utilizados para avaliar níveis de adaptação psicossocial positiva em face de eventos de vida importantes $^{(8)}$. Os escores têm uma amplitude que varia entre 25 a 175 pontos. Os escores de 25 a 120 indicam baixa resiliência, de 125 a 145 indicam moderadamente baixa a moderada resiliência e escores maiores que 145 indicam moderadamente alta a alta resiliência. Escala de Sobrecarga de Zarit: avalia aspectos relacionados à saúde do cuidador, e Inventário de Depressão de Beck: instrumento utilizado para rastreio de sintomas depressivos, todos traduzidos e validados para a cultura brasileira. As principais variáveis do estudo foram: nível de resiliência do cuidador, gênero, estado conjugal, escolaridade, idade, tempo de cuidado e grau de parentesco.

O tratamento estatístico dos dados foi realizado por meio de análises descritivas, Teste-t de Bonferroni, utilizado na comparação de médias dos grupos, testes de correlação Spearman, com a finalidade de verificar associação entre resiliência e as outras variáveis e regressão linear múltipla a fim de identificar os fatores que interferiram no nível de resiliência dos grupos. Para o estudo, foi adotado nível de significância 
de $5 \%$ e intervalo de $95 \%$ de confiança.

A amostra faz parte de uma casuística de ambulatório não sendo passível de generalizações dos resultados, devendo estes se limitarem apenas a população estudada.

\section{RESULTADOS}

As Tabelas 1 e 2 representam os dados sociodemográficos dos 66 cuidadores familiares que compuseram a amostra.

Tabela 1: Percentual de idosos por sexo em cada nível da classificação da doença de Alzheimer (DA), com medidas de dispersão e posição das variáveis Idade e Escolaridade por classificação da DA e considerando a população geral. São Carlos, SP, Brasil, 2014.

\begin{tabular}{cccccc}
\hline \multirow{2}{*}{ Sexo } & & DA Leve & DA Moderada & DA Avançada & DA Geral \\
\hline \multirow{4}{*}{ Idade } & Feminino & 12 & 12 & 16 & 40 \\
& Masculino & 13 & 10 & 3 & 26 \\
& Média & 55,1 & 56,3 & 56,7 & 56 \\
& Desvio-padrão & 12,7 & 15 & 12,8 & 13,3 \\
& Mínimo & 37 & 23 & 35 & 23 \\
\multirow{3}{*}{ Escolaridade } & Máximo & 80 & 85 & 88 & 88 \\
& Média & 6,9 & 3,9 & 4,4 & 8 \\
& Desvio-padrão & 3,6 & 3 & 3 & 3,9 \\
& Mínimo & 3 & 15 & 18 & 3 \\
\hline
\end{tabular}

Tabela 2: Frequência absoluta e relativa dos níveis das variáveis Estado Civil, Classe econômica e Grau de parentesco do cuidador por classificação da DA e população geral. São Carlos, SP, Brasil, 2014.

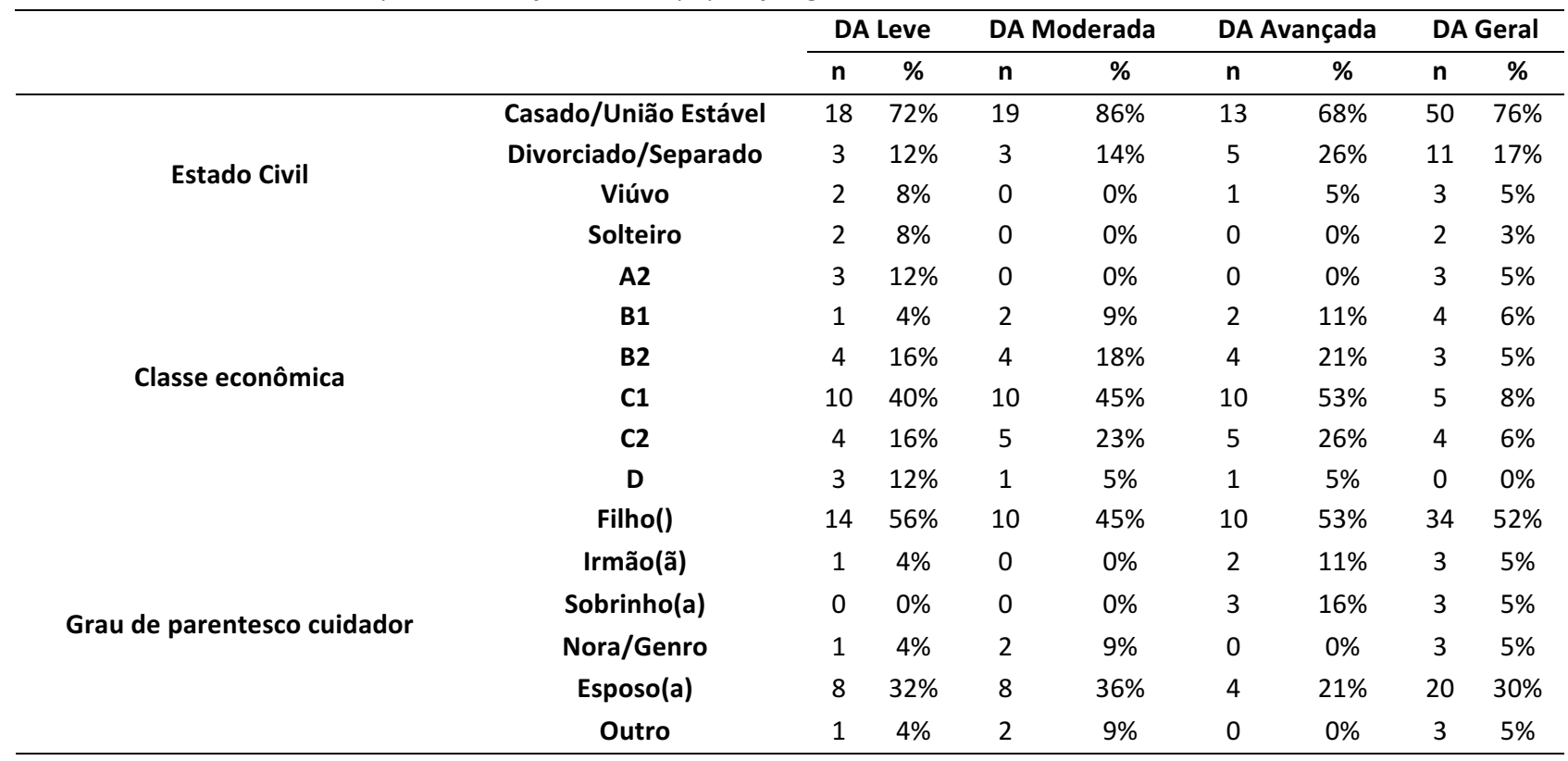

Em relação à pontuação na escala de resiliência, a maioria dos cuidadores (80,3\%), apresentou resiliência moderada. Apenas dois (4,2\%) tiveram nível de resiliência classificado como alto, e 11 cuidadores (23\%) apresentaram baixa resiliência.

De acordo com análise de regressão linear múltipla (Tabela 3), alguns fatores tiveram associação com o aumento da resiliência dos cuidadores, entre eles: a sobrecarga, os dias por semana que o cuidador passa 
com o idoso, grau de parentesco: quando o cuidador é nora/genro ou cônjuge em relação àqueles cuidadores que são filhos/as do idoso com DA, o fato de considerarem sua saúde física boa em relação àqueles que a consideram ótima e quando estes realizam outras atividades além do cuidado.

Tabela 3: Distribuição dos níveis de resiliência de cuidadores de idosos com doença de Alzheimer (DA), segundo a Escala de Resiliência $^{(8)}$. São Carlos, SP, Brasil, 2014.

\begin{tabular}{ccccccc}
\hline & \multicolumn{3}{c}{ Alta } & \multicolumn{2}{c}{ Moderada } & \multicolumn{2}{c}{ Baixa } \\
\cline { 2 - 7 } & $\mathbf{n}$ & $\mathbf{0}$ & $\mathbf{n}$ & $\mathbf{n}$ & $\mathbf{n}$ & \% \\
\hline DA leve & 0 & 0,0 & 21 & 84,0 & 4 & 16,0 \\
DA moderada & 1 & 4,5 & 17 & 77,3 & 4 & 18,2 \\
DA avançada & 1 & 5,3 & 15 & 79,0 & 3 & 15,7 \\
DA geral & 2 & 3,0 & 53 & 80,3 & 11 & 16,7 \\
\hline
\end{tabular}

Outros fatores se associaram com a diminuição dos níveis de resiliência dos cuidadores, sendo estes: autopercepção de saúde mental como sendo média, em relação aos cuidadores que consideraram sua saúde mental ótima, depressão, conforme o aumento desta os níveis de resiliência diminuíram, e a classe econômica, em que a resiliência diminuiu quando o cuidador pertencia a classe A2 em relação aos cuidadores das classes B1 e C1.

Tabela 4: Análise de regressão linear tendo como desfecho o grau de resiliência e as variáveis explicativas dos cuidadores de idosos com doença de Alzheimer. São Carlos, SP, Brasil, 2014.

\begin{tabular}{|c|c|c|c|c|}
\hline & & Estimativa & Estatística t & p-valor \\
\hline \multirow{3}{*}{ Variáveis } & Sobrecarga & 0,3 & 3,2 & 0 \\
\hline & Depressão & $-0,5$ & $-2,2$ & 0,03 \\
\hline & Idade & $-0,7$ & $-1,9$ & 0,07 \\
\hline \multirow{4}{*}{ Tempo de cuidado com idoso } & Horas por dia & $-0,3$ & $-1,8$ & 0,08 \\
\hline & Dia por semana & 5,6 & 3,1 & 0 \\
\hline & B1 & $-11,3$ & $-2,9$ & 0 \\
\hline & B2 & $-3,7$ & -1 & 0,28 \\
\hline \multirow[t]{5}{*}{ Classificação Econômica (A2) } & C1 & $-12,8$ & $-3,3$ & 0 \\
\hline & C2 & -8 & $-1,8$ & 0,07 \\
\hline & D & -18 & $-2,6$ & 0,01 \\
\hline & irmão & 6,5 & 1,1 & 0,07 \\
\hline & Sobrinho & 4,7 & 1 & 0,2 \\
\hline \multirow[t]{4}{*}{ Grau de parentesco } & Nora/genro & 46,7 & 4,7 & 0,32 \\
\hline & Cônjuges & 8,3 & 2 & 0 \\
\hline & Outro & 11,3 & 2,3 & 0,05 \\
\hline & Boa & 10,7 & 2,4 & 0,02 \\
\hline \multirow[t]{2}{*}{ Estado de Saúde } & Média & 5,1 & 0,9 & 0,37 \\
\hline & Ruim & $-0,2$ & 0 & 0,98 \\
\hline Realiza outras atividades & Sim & 4,4 & 2,2 & 0,03 \\
\hline
\end{tabular}

\section{DISCUSSÃO}

A maioria dos estudos encontrados apresenta concordância quanto ao gênero feminino dos cuidadores $^{(9-10)}$ e relacionam que, culturalmente, o cuidar é uma atividade essencialmente feminina e que diante de situações de fragilidade na estrutura familiar ou na presença de doença, o cuidado é absorvido pelas mulheres ${ }^{(11)}$. Entretanto, existe uma crescente participação do gênero masculino auxiliando no 
cuidado $^{(12)}$, o que justifica os $52 \%$ do gênero masculino no subgrupo DA leve, resultado desta pesquisa.

Em relação à idade dos cuidadores, em que se pontuou média de 56 anos, resultados semelhantes foram encontrados em estudos nacionais ${ }^{(12-13)}$, sendo que em alguns estudos internacionais foi encontrado média de idade de $69^{(14)}$ até 91 anos ${ }^{(15)}$. Essas diferenças reforçam o fato de que em países desenvolvidos a expectativa de vida é superior a dos países em desenvolvimento, justificando a maior média de idade dos cuidadores quando comparadas à média dos cuidadores encontradas nas pesquisas nacionais.

Um estudo nacional realizado com cuidadores familiares de idosos com $\mathrm{DA}^{(9)}$ também pontuou média de oito a 11 anos de escolaridade. No entanto, um outro realizado em Faro (Portugal) com 110 cuidadores informais de idosos dependentes apontaram nos seus resultados que a maioria dos cuidadores (67,3\%) possuíam quatro anos de estudo ou menos ${ }^{(16)}$, perfazendo um perfil de baixa escolaridade. Pessoas com nível de escolaridade mais baixo podem estar suscetíveis ao papel de cuidador, uma vez que a sociedade exige níveis mais elevados de educação para o mercado de trabalho formal ${ }^{(12)}$. Dessa forma, é compreensível que familiares com menos tempo de estudo se dediquem às tarefas domésticas e ao cuidado de seus familiares dependentes.

No que diz respeito ao estado conjugal dos cuidadores, a maioria se encaixa na categoria casado/a ou vive em regime de união estável, indo de encontro a literatura científica nacional e internacional, que trazem em seus resultados que a maioria das amostras estudadas também se encontrava na categoria casados ou união estável ${ }^{(12-13)}$.

Referente ao nível de resiliência apresentado pelos cuidadores, verificado por meio da ER, a presente pesquisa apontou que no grupo DA como um todo $(80,3 \%)$ e também seus subgrupos, a maioria dos cuidadores tiveram sua resiliência classificada como moderada. Vale destacar que apenas dois cuidadores tiveram pontuação para alta resiliência. O ser humano tem capacidade de adaptar-se as diversas situações, no entanto, cuidar de uma pessoa com doença crônica, sobretudo neurodegenerativa como a DA, pode repercutir no seu nível de resiliência.

Resultado semelhante foi evidenciado em um estudo internacional ${ }^{(17)}$ onde avaliaram a resiliência de 53 cuidadores de idosos com doença de Alzheimer. Em seus achados, pontuações mais altas de resiliência também se correlacionaram significativamente com a sobrecarga, com níveis de neuroticismo e extroversão, autoeficácia e autoestima, e menor uso de estratégias de enfrentamento focadas nas emoções.

Por meio de análise de regressão linear múltipla, foi verificado que algumas variáveis interferem no desfecho do nível de resiliência dos cuidadores, tanto para o aumento quanto para a diminuição destes níveis. No presente estudo, o grau de parentesco proporcionou um aumento de resiliência quando se tratou de cônjuges, nora ou genro e outros, contrapondo com a diminuição quando se tratou de filhos/as do idoso com DA. Resultados de outra pesquisa apontaram que filhos e cônjuges apresentaram médio e alto grau de resiliência quando comparados a irmãos e $\operatorname{cunhados}^{(9)}$. A presente pesquisa concorda com os dados desses mesmos autores, que trazem também que a boa percepção de saúde física teve associação com um elevado nível de resiliência. Os autores ressaltam ainda que o idoso com DA em seu contexto familiar e social poderá 
ter melhores condições de saúde e qualidade de vida se o cuidador tiver maior capacidade resiliente, pois este mantém o equilíbrio mental e físico, o que favorece a realização da sua tarefa de cuidar.

Cuidadores que realizavam outras atividades além do cuidado tiveram um aumento significativo em sua resiliência quando comparados àqueles que não realizavam. A esse respeito, outro estudo apontou sobre a necessidade de cuidadores desenvolverem atividades extras e observaram que cuidadores que participam em atividades da comunidade, de grupos de apoio e que tem suporte social enfrentam melhor sua função ${ }^{(10)}$. Isso nos chama a atenção para a importância do apoio de outros membros da família ao cuidador, ainda que este aconteça em momentos rápidos, de visitas informais. É relevante, ainda, que a equipe de saúde, ao atender o idoso com DA, insira também o cuidador no plano de cuidados, dizimando possíveis dúvidas acerca da patologia, contribuindo assim para diminuição de sua ansiedade e buscando estratégias para alívio de seu estresse.

Níveis mais altos de resiliência são relacionados com taxas mais baixas de depressão e melhor saúde física, e o apoio social tem sido um fator moderador da resiliência, pois uma variedade de tipos de apoio parece aliviar a sobrecarga física e mental causada pelo estresse ${ }^{(18)}$.

No presente estudo, todos os cuidadores eram familiares não remunerados e, além disso, tinham a carga emocional por serem integrantes da família, em constante exposição às demandas do doente.

Nesse sentido, o cuidador familiar necessita de orientações sobre como proceder nas situações do dia a dia, assim, como sempre que possível, receber visitas periódicas de profissionais da área da saúde, com a finalidade de sanar dúvidas acerca da patologia, conhecer os sintomas neuropsiquiátricos advindos dela nas fases mais avançadas, para que munidos de informações possam estar melhor preparados para o cuidado, assim como aliviar sua ansiedade referente ao desconhecido. Assim estarão menos propensos ao desgaste de cuidar, poderão ter melhor qualidade de vida e consequente preservação de sua saúde mental.

\section{CONCLUSÃO}

Este estudo permitiu concluir que cuidadores familiares de idosos com DA apresentam resiliência moderada, e que esta capacidade individual de enfrentamento sofre influências de determinadas variáveis. Estas podem contribuir para o aumento dessa capacidade, assim como agir na diminuição da mesma.

Uma lacuna identificada no estudo que merece ser destacada é a escassez de publicações de pesquisas quantitativas que abordam a temática resiliência, pois no Brasil verifica-se uma tendência à prevalência de estudos que abordam mais subjetivamente este tema. Isso dificultou em alguns aspectos a comparação dos resultados com outros estudos nacionais.

Estes achados poderão ser utilizados em áreas ligadas ao cuidado, e uma vez que foram identificadas variáveis que interferem na resiliência, estas poderão ser trabalhadas e melhoradas em benefício do cuidador e do paciente. 


\section{AGRADECIMENTOS}

A autora agradece à Coordenação de Aperfeiçoamento de Pessoal de Nível Superior (CAPES), pelo apoio na forma de bolsa de mestrado, possibilitando a realização desta pesquisa.

\section{REFERÊNCIAS}

1. Prince M, Bryce R, Albanese E, Wimo A, Ribeiro W, Ferri CP. The global prevalence of dementia: a systematic review and metaanalysis. Alzheimer's \& dementia: Alzheimers Dement. 2013;9(1):63-75e2.

2. McKhann, GM. at al. The diagnosis of dementia due to Alzheimer's disease: recommendations from the National Institute on Aging-Alzheimer's Association workgroups on diagnostic guidelines for Alzheimer's disease. Alzheimers Dement. 2011;7(3):263-9.

3. Alzheimer's Disease International. World Alzheimer Report 2009.[acesso em: 21 maio2013]. Disponível em: https://www.alz.co.uk/research/files/WorldAlzheimerReport.pdf.

4. Moreira, M. D.; Caldas, C. P. A importância do cuidador no contexto da saúde do idoso. Escola Anna Nery Revista de Enfermagem, Rio de Janeiro, v. 11, n. 3, p. 520-525, set. 2007.

5. Valente, L. et al. Autopercepção de saúde em cuidadores familiares e o tipo de demência: resultados preliminares de uma amostra ambulatorial. Revista Brasileira de Neurologia. 2013; 49(1): 13-19.

6. Reppold CT, Mayer JC, Almeida, LS, Hutz CS. Avaliação da resiliência: controvérsia em torno do uso das escalas. Psicol Reflex Crit. 2012;25(2):248-255.

7. Wagnild, GM, Young, HM. A Review of the Resilience Scale. Journal of Nursing Measurement, 2009; 17(2): 5-14.

8. Wagnild GM, Young HM. Development and psychometric evaluation of resilience scale. Journal of nursing measurement. 1993;1(2):165-178.

9. Gaioli CCLO, Furegato ARF, Santos JLF. Perfil de cuidadores de idosos com doença de Alzheimer associado à resiliência. Texto Contexto Enferm. 2012; 21(1):150-157.

10. Lopes SRA, Massineli CJ. Perfil e nível de resiliência dos cuidadores informais de idosos com Alzheimer. Aletheia. 2013; 40: 134-145.

11. Valente L,Truzzi A, Souza WF, Alves GS, Sudo FK,Alves CEO, et al. Autopercepção de saúde em cuidadores familiares e o tipo de demência: resultados preliminares de uma amostra ambulatorial. Rev Bras Neurol. 2013;49(1):13-9.

12. Gratão ACM, Vendrúscolo TRP, Talmelli LFS, Figueiredo LC, Santos JLF, Rodrigues RAP. Sobrecarga e desconforto emocional em cuidadores de idosos. Texto Contexto Enferm. 2012;21(2):304-312.

13. Bagne BM, Gasparino RC. Qualidade de vida do cuidador do portador de Doença de Alzheimer. Revista Enfermagem UERJ. 2014; 22(2): 258-263.

14. Haley W, Bergman EJ, Roth DL, McVie T, Gaugler JE, Mittelman MS. Long-Term Effects of Bereavement and Caregiver Intervention on Dementia Caregiver Depressive Symptoms. Gerontologist. 2008;48(6):732-740.

15. Lavretsky H, Siddarth P, Irwin MR. Improving depression and enhancing resilience in family dementia caregivers: a pilot randomized placebo-controlled trial of escitalopram. Am J GeriatrPsychiatry 2010 Feb; 18 (2): 154-64. 16. Rocha BMP, Pacheco JEP. Idoso em situação de dependência: estresse e coping do cuidador informal. Acta Paul Enferm. 2013; 26(1):50-6.

17. Fernández-Lansac V. Crespo-López M, Cáceres R, Rodríguez-Poyo M. Resiliencia en cuidadores de personas con demencia: estudio preliminar. Rev Esp Geriatr Gerontol.2012;47(3):102-9.

18. Dias R. Santos RL, Sousa MF, Nogueira MM, Torres B, Belfort T, et al. Resilience of caregivers of people with dementia: a systematic review of biological and psychosocial determinants. Trends Psychiatry Psychother. 2015;20(10):1-8. 